\title{
Simulation of Spatiotemporal Relationship between COVID-19 Propagation and Regional Economic Development in China
}

\author{
Dongya Liu ${ }^{1}$, Xinqi Zheng ${ }^{1, * \mathbb{C}}$ and Lei Zhang ${ }^{1,2}$ \\ 1 School of Information Engineering, China University of Geosciences, Beijing 100083, China; \\ dyliu@cugb.edu.cn (D.L.); zhanglei_2020@cugb.edu.cn (L.Z.) \\ 2 Key Laboratory of Urban Land Resources Monitoring and Simulation, Ministry of Natural Resources, \\ Shenzhen 518034, China \\ * Correspondence: zhengxq@cugb.edu.cn
}

check for updates

Citation: Liu, D.; Zheng, X.; Zhang,

L. Simulation of Spatiotemporal

Relationship between COVID-19

Propagation and Regional Economic

Development in China. Land 2021, 10,

599. https://doi.org/10.3390/

land10060599

Academic Editor: Rohan Bennett

Received: 15 April 2021

Accepted: 2 June 2021

Published: 5 June 2021

Publisher's Note: MDPI stays neutral with regard to jurisdictional claims in published maps and institutional affiliations.

Copyright: (c) 2021 by the authors. Licensee MDPI, Basel, Switzerland. This article is an open access article distributed under the terms and conditions of the Creative Commons Attribution (CC BY) license (https:// creativecommons.org/licenses/by/ $4.0 /)$.

\begin{abstract}
Coronavirus disease 2019 (COVID-19) propagation in 2019 posed serious threats and challenges to human public health and safety. Currently, COVID-19 is still not effectively controlled in certain countries and regions. This study combines the traditional susceptible-exposed-infectiousrecovered (SEIR) model, system dynamics (SD) model, and cellular automata (CA) model to construct a spatiotemporal dynamics pandemic model (SDPM). The SDPM is used to dynamically depict the spatiotemporal diffusion and outbreak of COVID-19 through research on the relationship between epidemic spread and regional development. The results show that: (1) There is a positive correlation between regional development and epidemic spread. The more developed the regional economy, especially in areas with short-range population migration from Hubei Province, the more severe the epidemic spread; and (2) The spatial isolation and control measures adopted by the government can effectively prevent the COVID-19 spread. The results explore the relationship between COVID-19 spread and regional economic development by studying the spatial and temporal transmission characteristics of COVID-19, and provide a scientific reference for the government to formulate reasonable response measures.
\end{abstract}

Keywords: COVID-19; SEIR model; system dynamics model; cellular automata model; spatiotemporal relationship

\section{Introduction}

In December 2019, the first patient with coronavirus disease 2019 (COVID-19) was diagnosed in Wuhan [1,2]. Wuhan is the capital of Hubei Province in China, and is well known as the "Nine Province Thoroughfares". Wuhan is the center of the Triangle of Central China and is one of the most important aviation, railway, and highway hubs in China. By the end of 2019, the permanent population of Wuhan reached 11.212 million [3]. Owing to the dual effects of the Spring Festival travel and Wuhan's particular economic and geographical situation, COVID-19 spread rapidly to other provinces in China and certain other countries and regions around the world. Wuhan announced the prohibition of people from leaving Wuhan on 23 January 2020, and lifted the spatial control measures on 8 April 2020 [4]. As of 0:00 on 8 April 2020, a total of 81,802 cumulative confirmed cases, 77,279 cumulative cured cases, and 3333 cumulative deaths had been reported according to the records from 31 provinces (autonomous regions and municipalities) and the Xinjiang Production and Construction Corps [5]. The cumulative deaths due to COVID-19 have far exceeded those due to severe acute respiratory syndrome (SARS) in 2003 [6].

COVID-19 is caused by severe acute respiratory syndrome coronavirus 2 (SARS-Cov2) [7]. The rapid propagation characteristic of COVID-19 poses a serious threat to human life and health [8]. Public health issues have become an important aspect of social security. Acute infectious diseases are widely and quickly spread by simultaneously infecting and affecting multiple individuals. COVID-19 transmission characteristics and development 
trends have attracted wide attention and research by domestic and foreign scholars [9-13]. Scholars have used a variety of methods to construct different epidemic simulation models to seek efficient and rapid preventive measures. Simulations can visually illustrate the rules for epidemic diffusion and enable the exploration of COVID-19 trends under various policy interventions. The simulation results can also provide a quantitative scientific basis and reference for the government, enabling those in power to take reasonable and effective preventive measures as soon as possible.

The susceptible-exposed-infectious-recovered (SEIR) model is a common infectious disease model that usually expresses a complex infectious disease system through multiple equations [14]. In the SEIR model, people are divided into four classes, namely: susceptible (referring to healthy people, who have a certain probability of being infected after contact with an infected person), exposed (referring to people who are infected by the infectious disease, but temporarily cannot propagate the disease to other people), infectious (referring to patients with infectious disease, who can transmit the disease to healthy people), and recovered (referring to patients who are cured) $[15,16]$. The relationships between these four classes are shown in Figure 1.

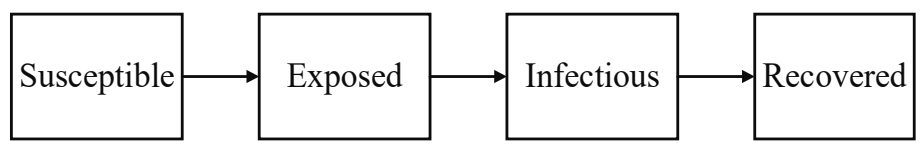

Figure 1. Traditional susceptible-exposed-infectious-recovered (SEIR) model.

There are two main problems with the traditional SEIR model: (1) It is assumed by default that convalescent patients carry antibodies for the infectious disease after being cured. That is, there will be no second infection in recovered patients. However, according to the relevant reports, certain COVID-19 survivors have been reinfected; that is, they can be diagnosed as COVID-19 patients again; and (2) The default-exposed person is partially converted into an infected patient, but the exposed person is not contagious. However, COVID-19 has also exhibited infectiousness during the incubation period. Therefore, the traditional SEIR model does not represent the transmission characteristics of the novel coronavirus.

Based on the above considerations, this study builds an extended SEIR-SD model based on the classical SEIR model and system dynamics (SD) model, which is used to simulate and predict the macro-quantity spread trends for COVID-19. The cellular automata (CA) model is used to construct an SEIR-CA model to simulate patients' spatiotemporal distribution. The CA model has been widely used in simulation studies of epidemics [17-19]. The CA model can easily express the spread relationship between patients and surrounding persons. Through simulation research on patients' distribution in time and space, the epidemic spread is explored at the micro-level. The spatiotemporal dynamics pandemic model (SDPM), which is based on the SEIR-SD-CA models, can reveal the propagation process of infectious diseases more intuitively and effectively. The model can also explore the laws of COVID-19 spread, both in macro and microscales of time and space. Section 2 introduces and builds the extended research methods. Section 3 describes the data used in this study, as well as presenting the simulation results and discussion. The conclusions are drawn in Section 4.

\section{SDPM}

\subsection{Extension of SEIR-SD Model}

The SD model mainly integrates system theory, cybernetics, and computer simulations. It dynamically explores the interaction and influence by constructing complex causal relationships between various elements. Through a combination of quantitative and qualitative methods, the SD model can be used to simulate the behavior and function of complex systems. In this work, an extended SEIR-SD model based on the principles of the traditional SEIR model and SD model was constructed. 
The relevant formulas in the traditional SEIR model can be expressed as Equations (1)-(5). The number of susceptible persons, $S_{t+1}$, at time $t+1$ can be expressed as:

$$
S_{t+1}=S_{t}-\frac{S_{t}}{N_{t}} \times \gamma \times I_{t} \times \beta,
$$

where $S_{t}$ reflects the number of susceptible persons (i.e., healthy people); $N_{t}$ represents the total population in the study area; $I_{t}$ is the number of infectious patients at time $t ; \gamma$ indicates the average number of persons contacted by infectious patients each day; and $\beta$ is the infection probability while a susceptible person is in contact with infectious patients.

The number of exposed persons, $E_{t+1}$, at time $t+1$ can be expressed as follows:

$$
E_{t+1}=E_{t}+\frac{S_{t}}{N_{t}} \times \gamma \times I_{t} \times \beta-\sigma \times E_{t},
$$

where $E_{t}$ is the number of exposed persons at time $t$; and $\sigma$ is the probability of an exposed person transforming into an infectious patient.

The number of infectious persons, $I_{t+1}$, at time $t+1$ can be expressed as:

$$
I_{t+1}=I_{t}+\sigma \times E_{t}-\omega \times I_{t},
$$

where $\omega$ is the probability of an infectious patient transforming into a recovered person.

The number of recovered persons, $R_{t+1}$, at time $t+1$ can be calculated as:

$$
R_{t+1}=R_{t}+\omega \times I_{t}
$$

where $R_{t}$ is the number of recovered persons at time $t$.

The total population $N_{t}$ in the study area at time $t$ can be written as:

$$
N_{t}=S_{t}+E_{t}+I_{t}+R_{t}
$$

There are two disadvantages of the traditional SEIR model, namely: (1) By default, it is assumed that the survivors carry antibodies for this type of infectious disease after being cured, which means the survivors will no longer be infected again; and (2) The default is that the exposed person is partially transformed into an infectious patient, but does not have infectiousness.

In this study, the SEIR model was extended as follows: (1) After infected persons are cured, they are automatically converted into healthy people and do not carry COVID-19 antibodies. Some recovered patients may still be reinfected and become exposed people again, and other patients become susceptible people, which means there is still the possibility of being infected; (2) Exposed persons are also infectious, and healthy people are also at risk of being infected after contact with exposed persons; and (3) Based on the original four classifications, death is added to the extended model. Some infected patients eventually die because they are incurable. The expanded relationship is shown in Figure 2.

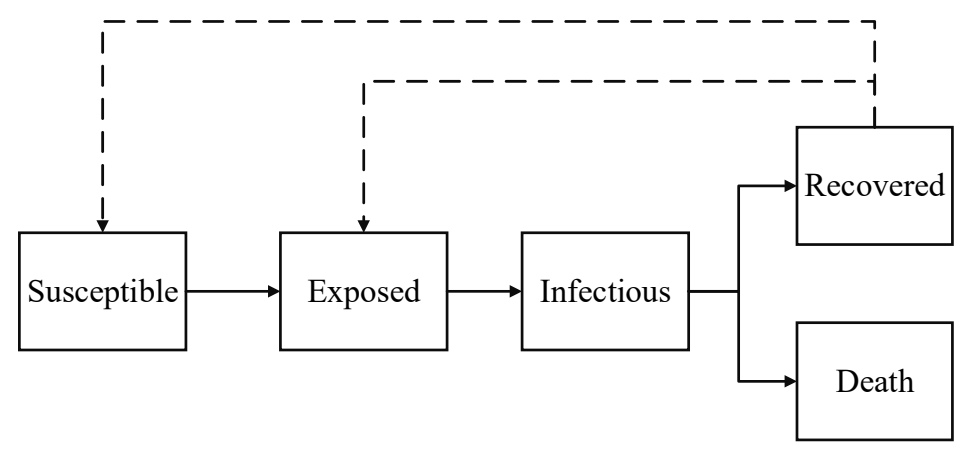

Figure 2. Extended SEIR model. 
The extended SEIR model relational expressions are expressed as Equations (6)-(11). The number of susceptible persons, $S_{t+1}$, at time $t+1$ can be extended as:

$$
S_{t+1}=S_{t}-\frac{S_{t}}{N_{t}} \times \gamma_{1} \times I_{t} \times \beta_{1}-\frac{S_{t}}{N_{t}} \times \gamma_{2} \times E_{t} \times \beta_{2}+(1-\varphi) \times R_{t},
$$

where $\gamma_{1}$ indicates the average number of persons in contact with infectious patients each day; $\gamma_{2}$ indicates the average number of persons contacted by exposed persons each day; $\beta_{1}$ is the infection probability while susceptible people contact infectious patients; $\beta_{2}$ is the probability of infected people contacting exposed persons; and $\varphi$ is the probability of recovered persons transforming into infected patients.

The number of exposed persons, $E_{t+1}$, at time $t+1$ can be extended as follows:

$$
E_{t+1}=E_{t}+\frac{S_{t}}{N_{t}} \times \gamma_{1} \times I_{t} \times \beta_{1}+\frac{S_{t}}{N_{t}} \times \gamma_{2} \times E_{t} \times \beta_{2}-\sigma \times E_{t}+\varphi \times R_{t},
$$

The number of infectious persons, $I_{t+1}$, at time $t+1$ can be extended as:

$$
I_{t+1}=I_{t}+\sigma \times E_{t}-\omega \times I_{t}-\mu \times I_{t},
$$

where $\mu$ is the death probability of infected patients.

The number of recovered persons, $R_{t+1}$, at time $t+1$ can be calculated as:

$$
R_{t+1}=R_{t}+\omega \times I_{t}-\varphi \times R_{t},
$$

The number of deaths, $D_{t+1}$, at time $t+1$ can be expressed as:

$$
D_{t+1}=\mu \times I_{t},
$$

The total population, $N_{t}$, in the study area at time $t$ can be written as:

$$
N_{t}=S_{t}+E_{t}+I_{t}+R_{t}+D_{t},
$$

According to Equations (6)-(11), an SEIR-SD-CA causality loop diagram is constructed for COVID-19 (Figure 3), which is used to conduct dynamic simulation research on the epidemic situation.

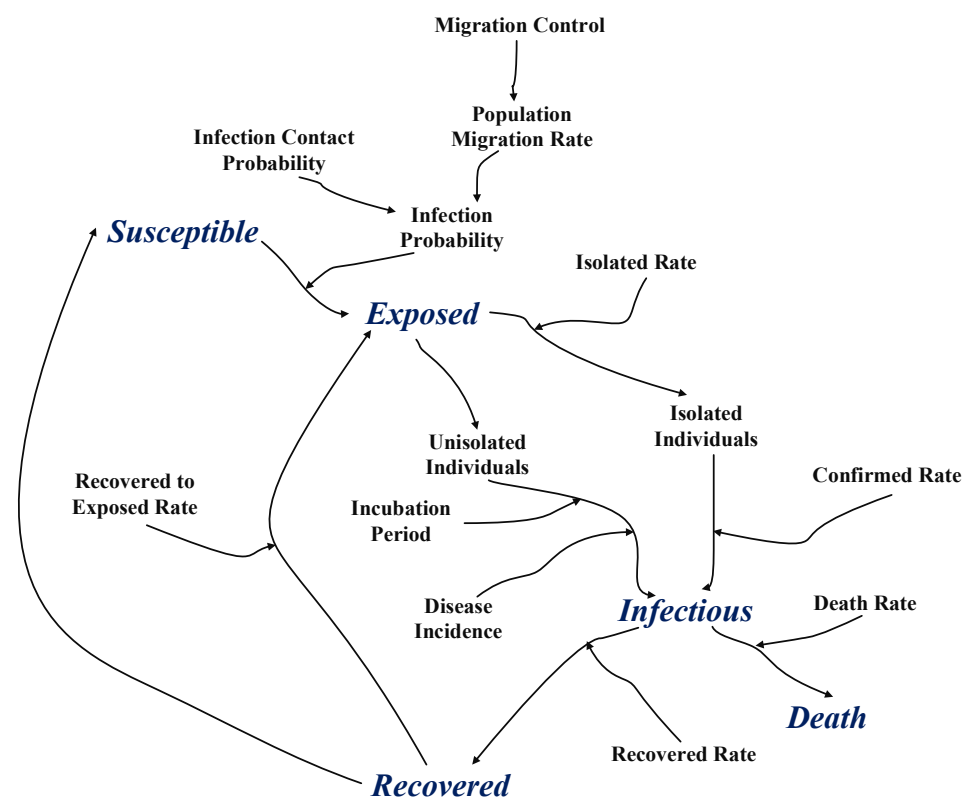

Figure 3. Dynamic causality diagram for the extended SEIR-SD model. 


\subsection{Rules of the SEIR-CA Model}

The CA model originates from complex science [20] and is a bottom-up microscopic dynamic model that is discrete in both time and space [19]. The CA model has the ability to simulate the spatiotemporal evolution of complex systems and reveals macroscopic complex phenomena through the evolutionary behavior of each cell on the microscopic unit. There are five basic elements in the CA model: cell, cell state, cell space, neighborhood, and transition rule.

(1) The cell is the most basic and smallest unit in the CA model and is the object of model research.

(2) The cell state is a series of data used to represent the cell's current attributes. Each cell has only one state at a certain time.

(3) The cell space is the collection of spatial network points where the cells are located. In this study, the cell space refers to the scope of the study area.

(4) The neighborhood consists of the other cells within a certain range around the central cell and is an important component of the CA model. Common neighborhoods include the Von Neumann neighborhood and Moore neighborhood. The Von Neumann neighborhood includes four basic adjacent cells, and the Moore neighborhood includes eight adjacent cells [21].

(5) The transition rule is the core element of the CA model. Based on the current cell state and the current neighborhood cell state, the next moment cell state is determined by the transition function. The traditional CA transition function is:

$$
S_{t+1}=f\left(S_{t}, N_{t}\right),
$$

where $S_{t+1}$ represents the cell state at time $t+1 ; S_{t}$ is the cell state at time $t$; and $N_{t}$ refers to the neighborhood cell state at time $t$.

In this study, based on COVID-19 propagation characteristics, the CA transition rule is extended as:

$$
S_{t+1}=f\left(S_{t}, N_{t}, \text { Pop }_{t}, G D P_{t}, \text { Migw }_{t}, \text { Migh }_{t}, \text { Ran }_{t}\right),
$$

where $P o p_{t}$ represents the population distribution at time $t ; G D P_{t}$ is the economic development at time $t ; M i g w_{t}$ indicates the population migration from Wuhan to the cell at time $t ; \mathrm{Migh}_{t}$ is the population migration from Hubei Province to the cell at time $t$; and Rant represents sudden random conditions at time $t$.

The above relationship can be expressed as:

$$
P_{t}=\sum_{i=1}^{8} N_{i, t} \times P_{p o p, t} \times P_{G D P, t} \times P_{M i g w, t} \times P_{M i g h, t} \times P_{R a n, t},
$$

where $\sum_{i=1}^{8} N_{i, t}$ is the influence probability of the neighborhood cells at time $t ; P_{p o p, t}$ is the influence probability of the population distribution at time $t ; P_{G D P, t}$ is the probability of impact on economic development at time $t ; P_{M i g w, t}$ is the influence probability of population migration from Wuhan to the target cell at time $t ; P_{M i g h, t}$ represents the probability of population migration from Hubei Province to the target cell at time $t$; and $P_{R a n, t}$ indicates the probability of random events at time $t$.

\subsection{Model Implementation}

The SDPM was constructed and implemented based on the NetLogo platform and Logo language. NetLogo was first developed by Northwestern University in 1999 [22] and is a free and open-source software package that has been continuously updated. NetLogo can build an easy-to-operate graphical user interface and implement module functions based on a specific programming language, which is highly suitable for building complex and dynamic models.

The SDPM built on NetLogo has a friendly interface that can be conveniently operated (as shown in Figure 4). In this model, various simulation scenarios can be automatically simulated and run by inputting the initial operating parameters. Moreover, changes in 
the epidemic distribution can be dynamically monitored in real time, as well as dynamic changes in macro data. For map display data, the model outputs data in standard ASCII format, which can be easily imported into ArcGIS and other software for further data analysis and mining. For macro statistics, the model outputs data in a standard CSV format. The output of the standard data results provides a basis for further research.

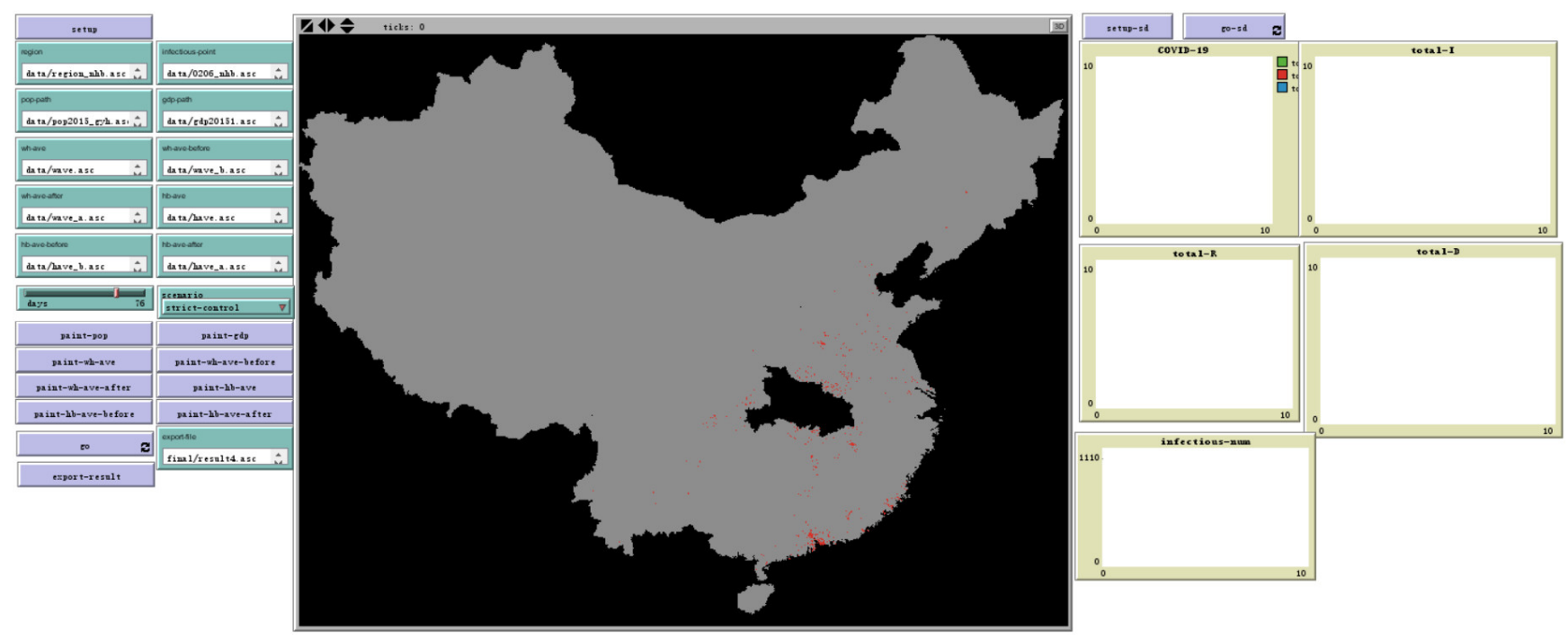

Figure 4. Spatiotemporal dynamics pandemic model (SDPM) user interface.

\section{Results}

\subsection{Study Area and Data}

In this study, we used COVID-19-related national statistics data (excluding Hong Kong, Macao, and Taiwan) from 23 January 2020 (when Wuhan announced a measure to forbid departure from Wuhan) [23] to 8 April 2020 (when the government lifted the travel limit measures) [24]. The data included cumulative confirmed cases, existing confirmed cases, daily increased confirmed cases, cumulative recovered cases, daily increased recovered cases, cumulative deaths, daily increased deaths, cumulative suspected cases, existing suspected cases, daily increased suspected cases, cumulative close contacts, existing medical observers, and daily medical observers (Figure 5). These data were obtained from the National Health Commission of the People's Republic of China.

In addition, we obtained the migration rates from Wuhan to other prefecture-level cities (excluding Hong Kong, Macao, and Taiwan), and the migration rates from Hubei Province to other prefecture-level cities (excluding Hong Kong, Macao, and Taiwan). The data were spatially vectorized and obtained from the Baidu epidemic real-time big data report (https:/ / voice.baidu.com/act/newpneumonia/newpneumonia, accessed on 8 April 2020). In this study, the average level of population migration from Wuhan to other prefecture-level cities (excluding Hong Kong, Macao, and Taiwan) before Wuhan's closure of all channels was obtained, as well as the average level of population migration from Hubei Province to other prefecture-level cities (excluding Hong Kong, Macao, and Taiwan) before and after the Spring Festival travel season, respectively (Figure 6). 


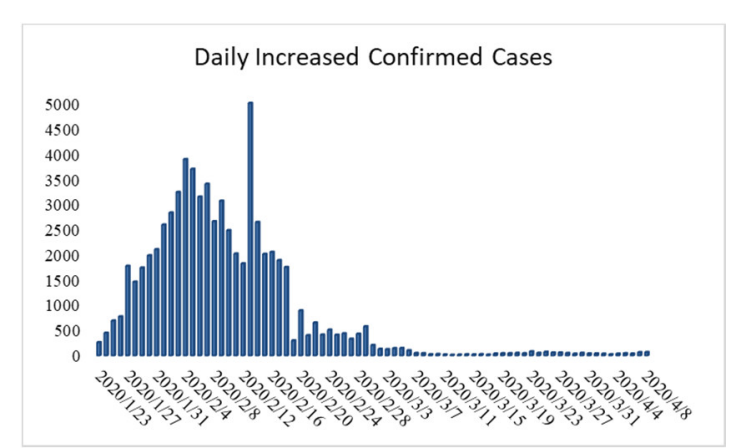

(a)

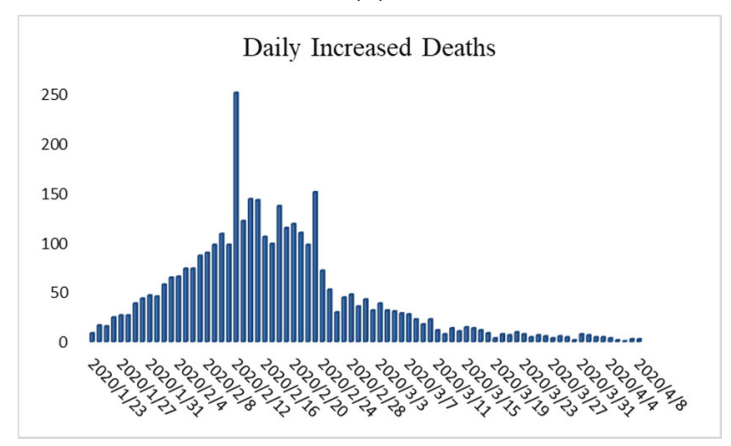

(c)

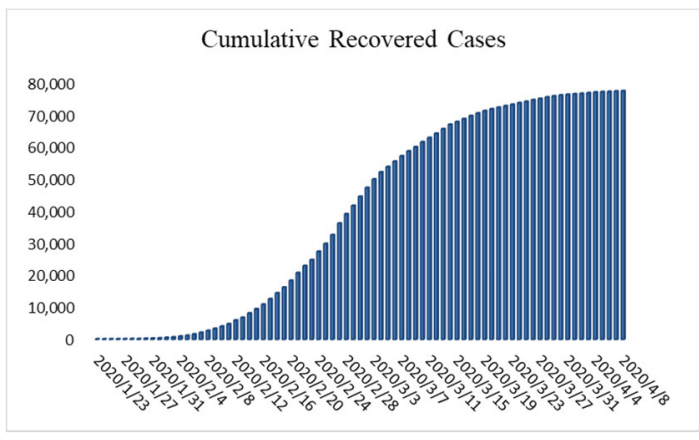

(e)

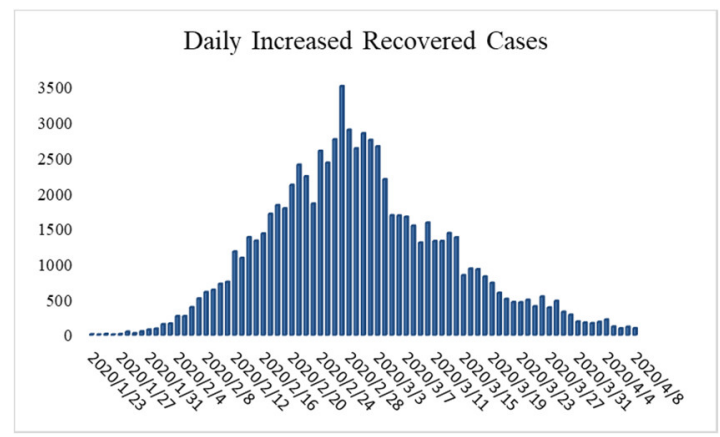

(b)

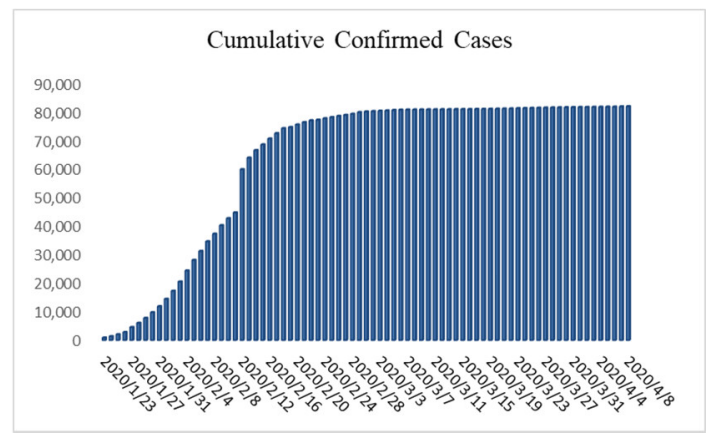

(d)

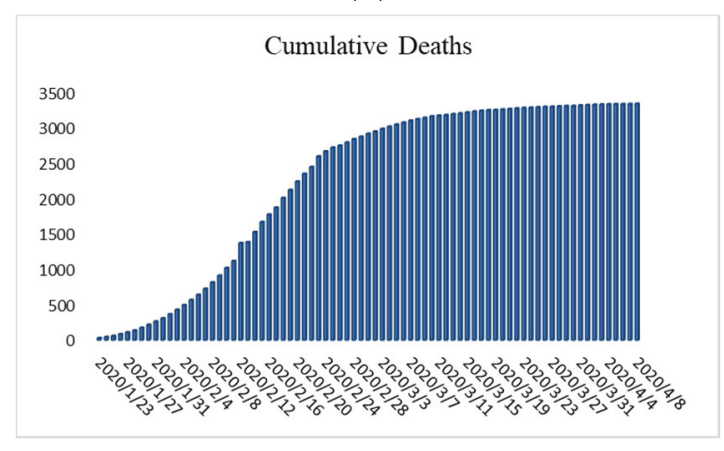

(f)

Figure 5. Number of daily increased and cumulative cases for confirmed cases, recovered cases, and deaths. (a) Daily increased confirmed cases; (b) daily increased recovered cases; (c) daily increased deaths; (d) cumulative confirmed cases; (e) cumulative recovered cases; (f) cumulative deaths.

The spatial location data of daily COVID-19 patients were collected in this study from Tencent Kandian (https:/ / ncov.html5.qq.com/community, accessed on 20 March 2020) and Nandu Media (https://m.mp.oeeee.com/h5/pages/v20/nCovcase/, accessed on 20 March 2020). The national GDP and population spatial distribution data were obtained from the Resource and Environment Data Cloud Platform (http:/ / www.resdc.cn/Default. aspx, accessed in 2015) [25]. Detailed data are shown in Table 1.

In this study, all spatial data were unified into the Universal Transverse Mercator (UTM) coordinate system. Moreover, to eliminate the influence of different dimensions, the migration rate, population distribution data, and GDP distribution data were normalized according to the following normalization equation [26,27]:

$$
S_{i}^{\prime}=\left\{\begin{array}{c}
\frac{S_{i}-\min \left(S_{i}\right)}{\max \left(S_{i}\right)-\min \left(S_{i}\right)}, \text { if } \max \left(S_{i}\right) \neq \min \left(S_{i}\right) \\
1, \text { if } \max \left(S_{i}\right)=\min \left(S_{i}\right)
\end{array}\right.
$$


where $S_{i}^{\prime}$ is the value after normalization; $S_{i}$ is the value before normalization; $\min \left(S_{i}\right)$ is the minimum of all values; and $\max \left(S_{i}\right)$ is the maximum of all values.

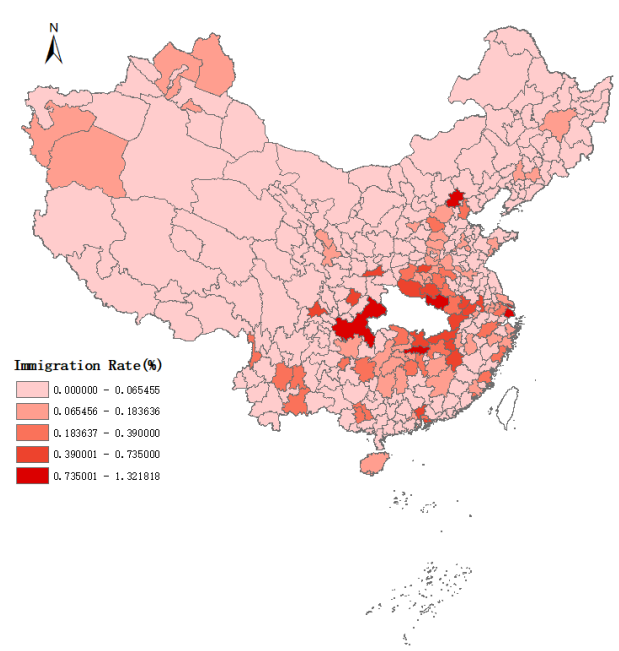

(a)

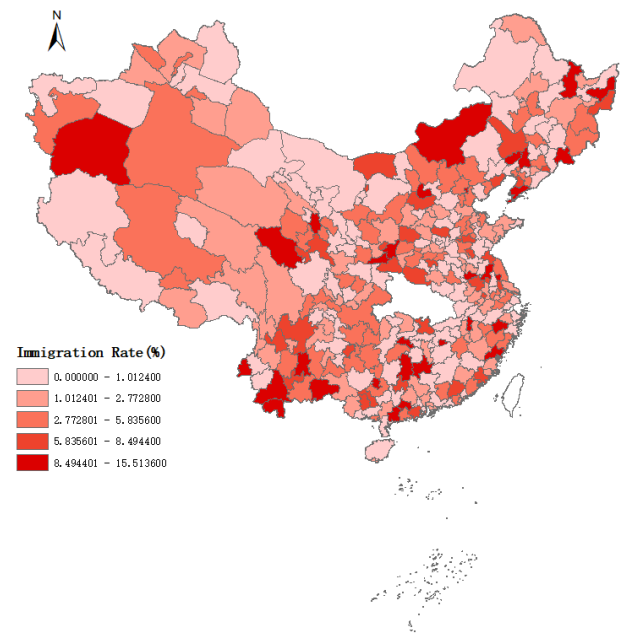

(b)

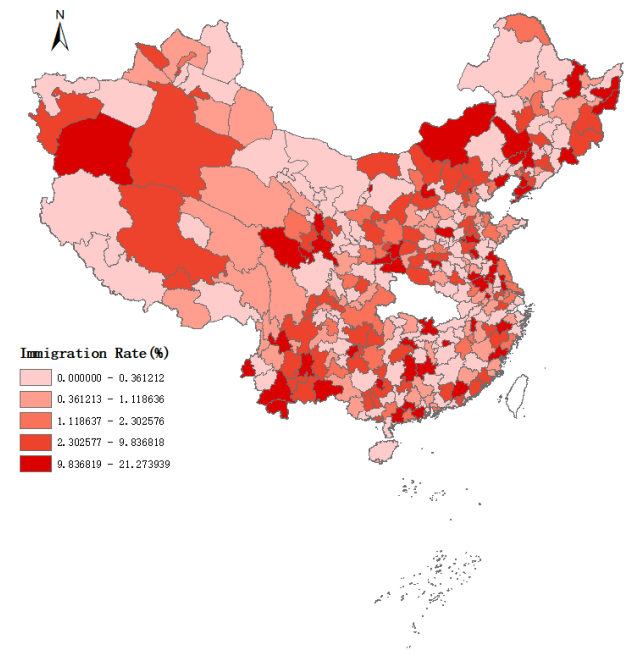

(c)

Figure 6. Population migration rates from Wuhan and Hubei Province to other prefecture-level cities (excluding Hong Kong, Macao, and Taiwan), respectively. (a) Migration rates from Wuhan to other prefecture-level cities before Wuhan's closure of all channels; (b) migration rates from Hubei Province to other prefecture-level cities before Spring Festival; (c) migration rates from Hubei Province to other prefecture-level cities after Spring Festival. 
Table 1. Data used in the study.

\begin{tabular}{ccc}
\hline Data & Data type & Data source \\
\hline $\begin{array}{c}\text { Macro epidemic statistics data } \\
\text { (such as cumulative confirmed } \\
\text { cases, cumulative cured cases) } \\
\begin{array}{c}\text { Migration rate from Wuhan to } \\
\text { other prefecture-level cities } \\
\text { (except Hong Kong, Macao, } \\
\text { and Taiwan) }\end{array}\end{array}$ & Continuous variable & $\begin{array}{c}\text { National Health Commission of } \\
\text { the People's Republic of China }\end{array}$ \\
$\begin{array}{c}\text { Migration rate from Hubei } \\
\text { Province to other prefecture-level } \\
\text { cities (excluding Hong Kong, } \\
\text { Macao, and Taiwan) }\end{array}$ & Continuous variable & $\begin{array}{c}\text { Baidu epidemic real-time } \\
\text { big data report }\end{array}$ \\
$\begin{array}{c}\text { Patients spatial location data } \\
\text { Population spatial } \\
\text { distribution data }\end{array}$ & Shape & $\begin{array}{c}\text { Baidu epidemic real-time } \\
\text { big data report }\end{array}$ \\
GDP spatial distribution data & Shape & $\begin{array}{c}\text { Tesource and Environment Data } \\
\text { Cloud Platform } \\
\text { Median, and Nandu }\end{array}$ \\
\hline
\end{tabular}

\subsection{Simulation Results of COVID-19 Propagation in Macro Quantity}

In the SEIR-SD model, the time was set to $1 \mathrm{~d}$ (one day). The values of the parameters were determined by references [28-31] and the law of historical data development. The specific values are presented in Table 2.

Table 2. Details of parameters.

\begin{tabular}{ccc}
\hline Parameters & Values of Parameters & Source \\
\hline$\gamma_{1}$ & 3.22 & Estimated and [28-30] \\
$\beta_{1}$ & $7.33 \%$ & Estimated and [28-30] \\
$\gamma_{2}$ & 3.22 & Estimated and [28-30] \\
$\beta_{2}$ & $7.33 \%$ & Estimated and [28-30] \\
$\varphi$ & $12.16 \%$ & [31] \\
$\sigma$ & 0.5 & Estimated \\
$\omega$ & 0.062 & Calculated \\
$\mu$ & 0.004 & Calculated \\
\hline
\end{tabular}

Based on the SEIR-SD model, we simulated the trend of cumulative confirmed cases, cumulative cured cases, and cumulative death cases with respect to the time from 23 January 2020 to 8 April 2020 (Figure 7). Through a visual comparison, it can be seen that the simulation results are relatively close to the real data. Simultaneously, we quantitatively calculated the simulation precision (as shown in Figure 8). It can be seen from Figure 8 that, in addition to some deviations in the simulation effect of the cumulative recovered cases, the cumulative confirmed cases and cumulative death cases both achieved good simulation results. 


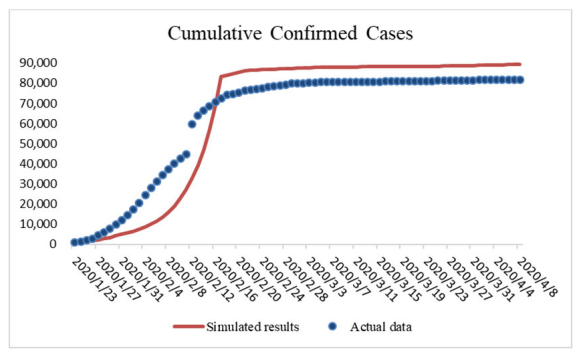

(a)

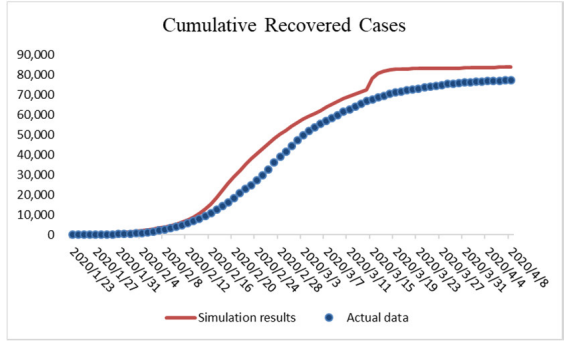

(b)

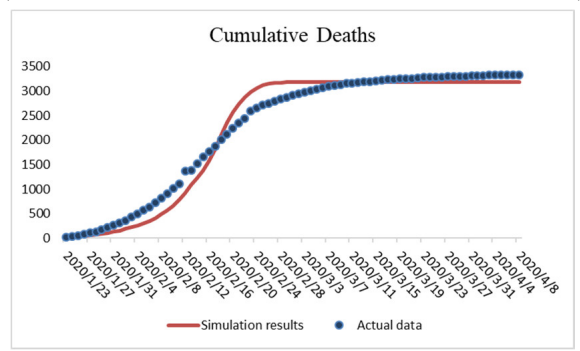

(c)

Figure 7. Epidemic cumulative cases simulation from 23 January 2020 to 8 April 2020. (a) Comparison of cumulative confirmed cases; (b) comparison of cumulative recovered cases; (c) comparison of cumulative deaths.

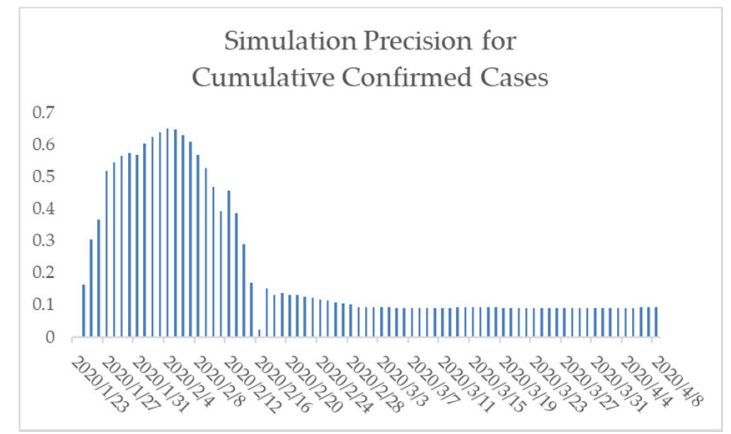

(a)

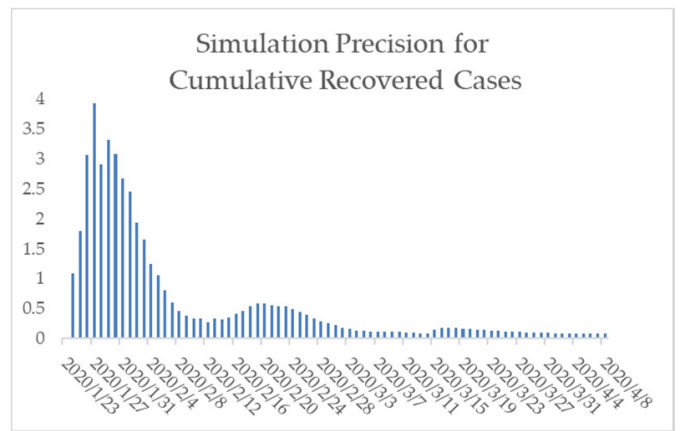

(b)

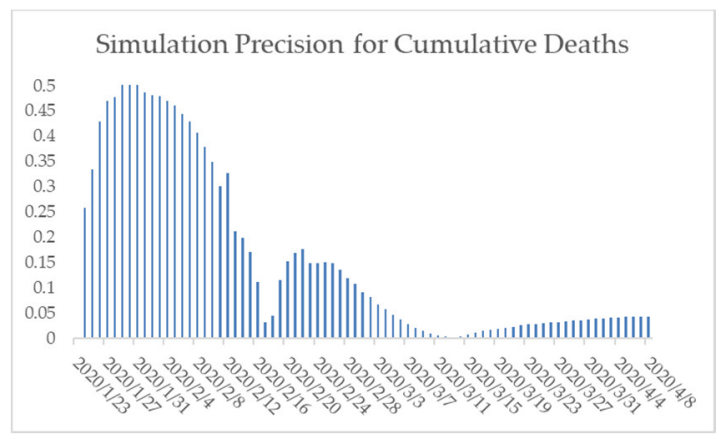

(c)

Figure 8. Epidemic cumulative cases simulation precision from 23 January 2020 to 8 April 2020. (a) Simulation precision for cumulative confirmed cases; (b) simulation precision for cumulative recovered cases; (c) simulation precision for cumulative deaths.

It can be seen from the figures that the epidemic has been basically under control since March in China. The number of confirmed cases and death cases did not increase significantly. Moreover, with the continuous investment in medical staff, medical facilities, and construction of cabin hospitals, the cure rate has been increasing, while the death rate has gradually decreased. 


\subsection{Scenario Simulations for COVID-19 Spatial Propagation}

In this study, three different simulation scenarios were considered: (1) according to the current development trend, the government adopted a certain degree of interference measures, such as traffic control; (2) the epidemic spread freely, that is, the government did not take any spatial control measures; and (3) the authorities took measures to further strengthen urban management and control of Wuhan and even the entire Hubei Province.

The cell state is the person's state who is in the cell, namely: susceptible, exposed, infectious, and recovered. This study only considers the research on the COVID-19 spatial spread; that is, for the time being, only the transition from a healthy person to a confirmed patient is considered, whereas the healing cell state and death cell state are not taken into account. Additionally, owing to the limitation of data acquisition, we only obtained the spatial location data for certain confirmed patients from 6 February 2020 to 20 March 2020. Therefore, only some sample data were used to approximately estimate the COVID-19 spatial spread trend.

\subsubsection{Scenario 1: Current Development Trend}

Based on the space control measures taken by the government at this stage, the epidemic spread trend in the country (excluding Hong Kong, Macao, and Taiwan) centered on Hubei Province was explored. The simulation results are presented in Figure 9. It can be seen from the figure that the epidemic is centered on Hubei Province, and gradually spread to surrounding provinces, especially in areas close to Hubei Province, such as Beijing, the Pearl River Delta, and the Yangtze River Delta. The spatial traffic control measures implemented by the government have significantly prevented the spread of the epidemic to more cities and regions, and effectively limited the speed and scope of COVID19 propagation. Owing to the limitation of data acquisition, the real data we obtained at each time point are sample points data. At the same time, different time points cannot guarantee that the sample points are the same, such that accurate calculations cannot be performed. However, we can see through the visual comparison method that the spatial distribution of simulated data and real data on 20 March 2020 is essentially the same.

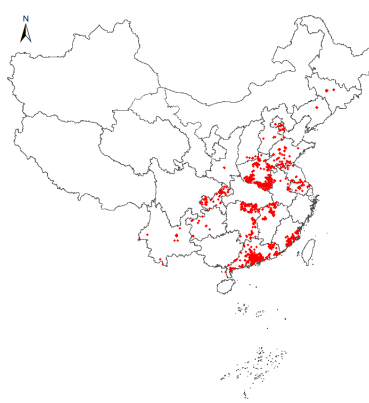

(a)

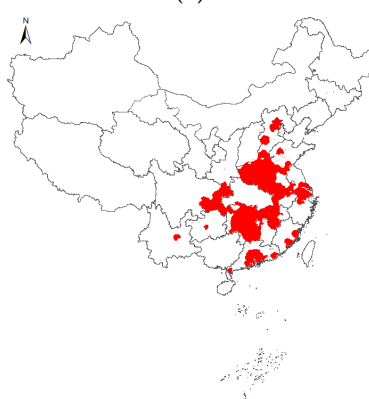

(d)

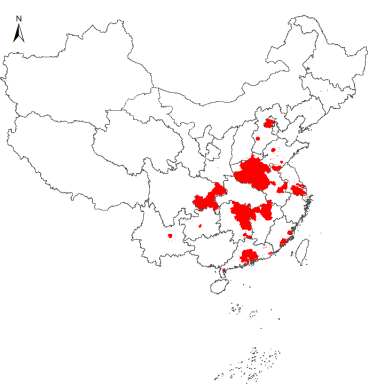

(b)

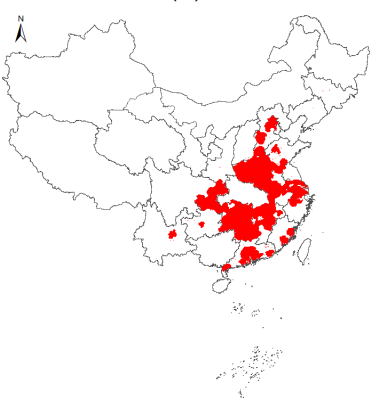

(e)

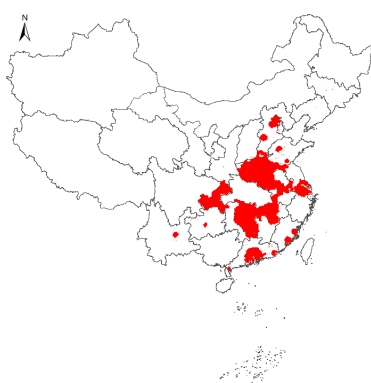

(c)

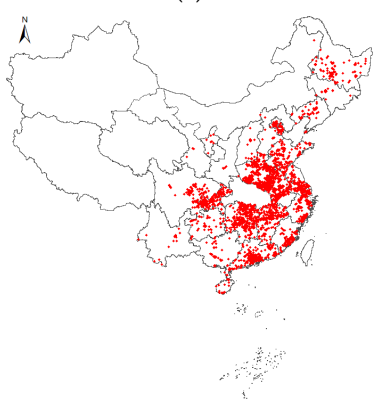

(f)

Figure 9. COVID-19 spread under current trends. (a) 6 February 2020 (actual data); (b) 16 February 2020 (simulated data); (c) 26 February 2020 (simulated data); (d) 7 March 2020 (simulated data); (e) 20 March 2020 (simulated data); (f) 20 March 2020 (actual data). 


\subsubsection{Scenario 2: Free Diffusion}

Under this scenario, assuming that the authorities do not take any effective control measures, people flow freely. Population migration was calculated based on the migration rate prior to the closure of Wuhan. The epidemic spread effect is shown in Figure 10.

It can be seen from Figure 10 that, in the context of no government intervention, the epidemic spreads rapidly across the country. Combining these results with Figure 6 (the migration rate from Wuhan and Hubei Province to other prefecture-level cities), it is observed that in the provinces and cities that experienced short-range migration from Wuhan, there gradually appeared confirmed patients and spread to other prefecture-level cities, especially the Xinjiang Uygur Autonomous Region, Inner Mongolia Autonomous Region, and Three Northeastern Provinces.

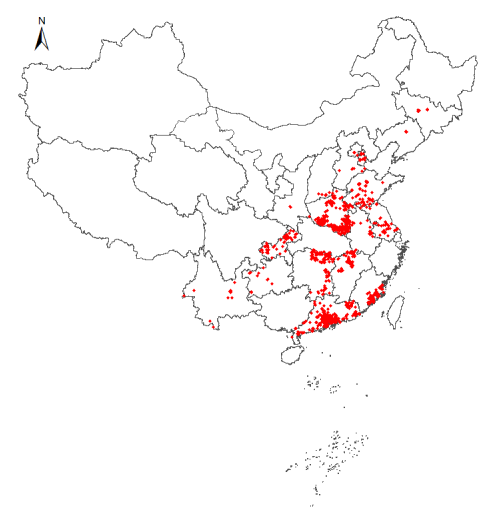

(a)

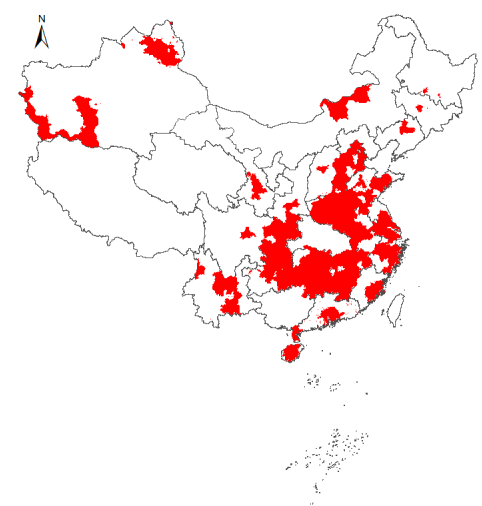

(d)

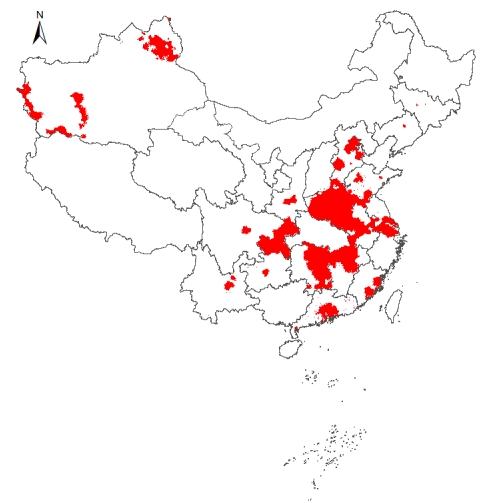

(b)

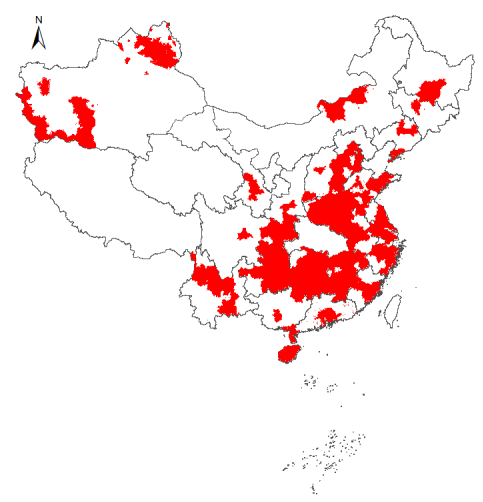

(e)

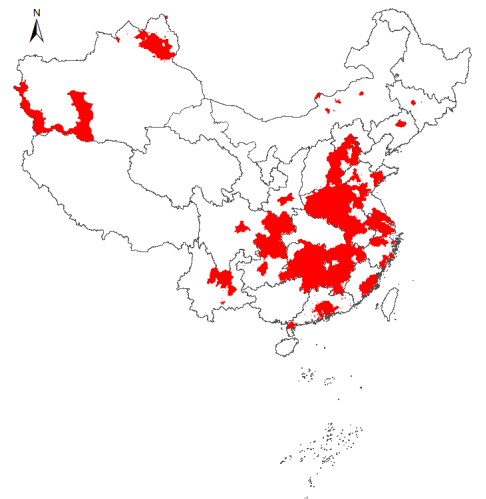

(c)

Figure 10. COVID-19 spread under no government control. (a) 6 February 2020 (actual data); (b) 16 February 2020 (simulated data); (c) 26 February 2020 (simulated data); (d) 7 March 2020 (simulated data); (e) 20 March 2020 (simulated data).

\subsubsection{Scenario 3: Tighter Control Measures}

Assuming that at the beginning of the epidemic, the government takes space isolation control measures to prohibit unnecessary movement of people, the epidemic spread is shown in Figure 11.

Under these control measures, the epidemic is controlled to a great extent. The epidemic mainly propagates in neighboring provinces and economically developed areas around Hubei Province, but does not spread in other provinces and regions across the country. 


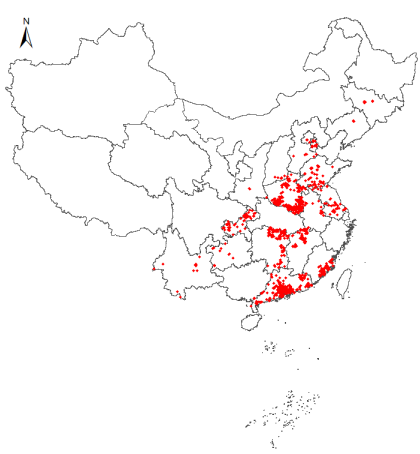

(a)

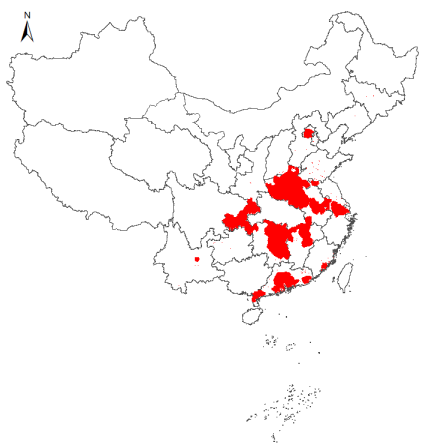

(d)

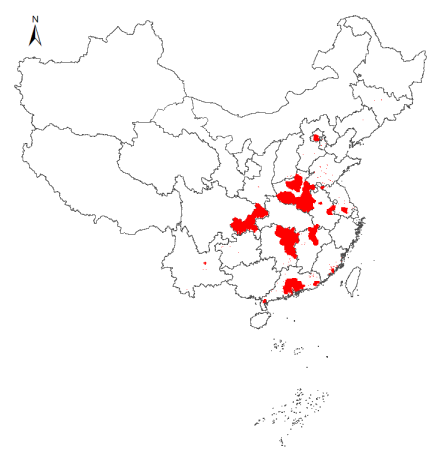

(b)

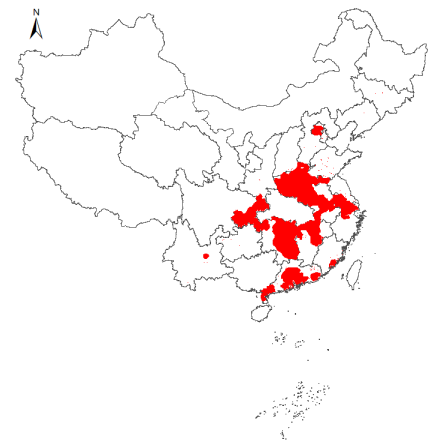

(e)

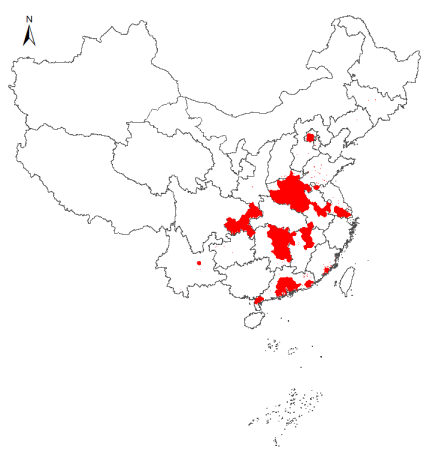

(c)

Figure 11. COVID-19 spread under tighter control measures. (a) 6 February 2020 (actual data); (b) 16 February 2020 (simulated data); (c) 26 February 2020 (simulated data); (d) 7 March 2020 (simulated data); (e) 20 March 2020 (simulated data).

\subsubsection{Discussion}

In this study, we calculated the macro-statistical data for the partial spatial distribution of confirmed patients in the above three scenarios. Figure 12 shows the results of the comparison. It is obvious that, from 6 February 2020 to 20 March 2020, the number of confirmed patients increased to 38,684 according to the current trend. Without any government intervention, which means that personnel can naturally flow between provinces and cities, the number increased to 85,007 , which is more than twice the number under Scenario 1. If in the early stage of the epidemic the authorities took traffic control measures to prohibit the free movement of people in Wuhan and Hubei Province, the number of confirmed patients would be 28,258 , which would greatly control the epidemic spread.

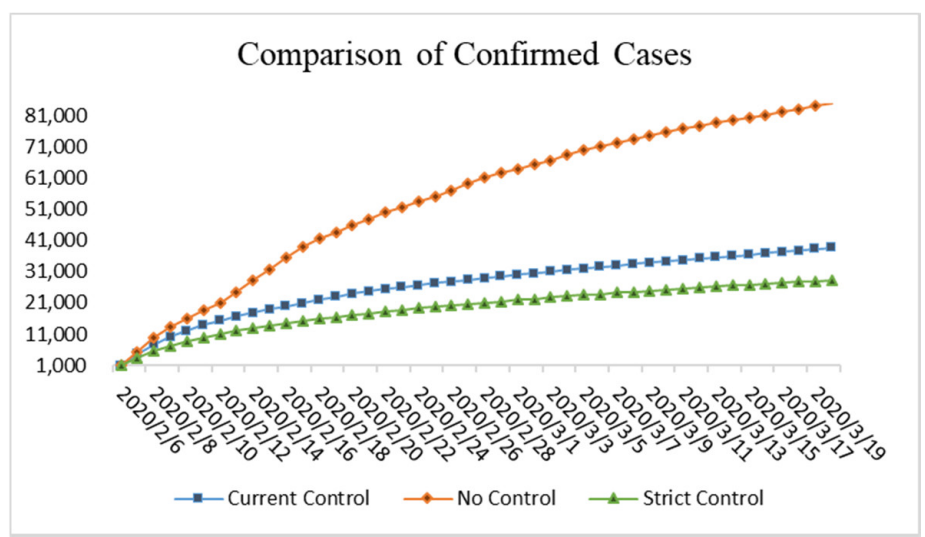

Figure 12. Comparison of partially confirmed patients under the above three scenarios. 


\section{Conclusions}

Based on the SD model and the CA model, the SDPM is proposed by extending the traditional SEIR model. This model can dynamically simulate the macroscopic quantitative change characteristics of COVID-19 over time, such as cumulative confirmed patients, cumulative cured cases, and cumulative deaths. Meanwhile, the model can also describe the spread of COVID-19 in space. The model realized the multi-angle and multi-dimensional real-time dynamic tracking and description of COVID-19 both in time and space and at macro and micro scales.

By exploring the relationship between the epidemic transmission characteristics and regional development power in time and space, it was found that the more developed the region, the more serious the epidemic spread. It was also shown that the greater the population migration from Wuhan and Hubei Province, the more severe were the effects of the epidemic. At the same time, in this study, three different simulation scenarios regarding the government's spatial traffic control were established. The results showed that the government's spatial traffic control measures, the prohibition of unnecessary movement of people, and the timeliness of control measure implementation will largely curb the spread of the epidemic to other regions.

COVID-19 propagation is complex and variable, with many influencing factors. In this study, due to the limitations of data acquisition, only certain confirmed patients' spatial distribution data were used for simulation research, and the acquisition time was only from 6 February 2020 to 20 March 2020. If it were possible to acquire longer time series and more large-scale spatial data of confirmed patients, the results would be more accurate and effective for obtaining epidemic spread characteristics.

Author Contributions: Conceptualization, D.L.; methodology, D.L. and X.Z.; software, D.L.; data curation, D.L. and L.Z.; writing—original draft preparation, D.L.; writing-review and editing, D.L. All authors have read and agreed to the published version of the manuscript.

Funding: This research was supported by the Open Fund of Key Laboratory of Urban Land Resources Monitoring and Simulation, Ministry of Natural Resources (No. KF-2020-05-063) and the Fundamental Research Funds for the Central Universities (No. 2652019001, No. 2652020004).

Institutional Review Board Statement: Not applicable.

Informed Consent Statement: Not applicable.

Data Availability Statement: Not applicable.

Acknowledgments: We thank all the medical staff who are involved in COVID-19 patient treatment; we thank the National Health Commission of the People's Republic of China for providing the data used in this study; and we acknowledge all websites that collect and share COVID-19 data.

Conflicts of Interest: The authors declare no conflict of interest.

\section{References}

1. Huang, C.; Wang, Y.; Li, X.; Ren, L.; Zhao, J.; Hu, Y.; Zhang, L.; Fan, G.; Xu, J.; Gu, X. Clinical features of patients infected with 2019 novel coronavirus in Wuhan, China. Lancet 2020, 395, 497-506. [CrossRef]

2. Li, Q.; Guan, X.; Wu, P.; Wang, X.; Zhou, L.; Tong, Y.; Ren, R.; Leung, K.S.; Lau, E.H.; Wong, J.Y. Early transmission dynamics in Wuhan, China, of novel coronavirus-infected pneumonia. N. Engl. J. Med. 2020, 382, 1199-1207. [CrossRef] [PubMed]

3. Wuhan Statistics Bureau. 2019 Statistical Communique on Wuhan's National Economic and Social Development Wuhan Statistics Bureau. Available online: http://tj.wuhan.gov.cn/tjfw/tjgb/202004/t20200429_1191417.shtml (accessed on 29 March 2020).

4. The People's Government of Hubei Province. Notice of Hubei Province Headquarters for COVID-19 Prevention and Control. Hubei Province Headquarters for COVID-19 Prevention and Control. Available online: https: / / baijiahao.baidu.com/s?id=1663 322708039658101\&wfr=spider\&for $=$ pc (accessed on 7 April 2020).

5. National Health Commission of the People's Republic of China. The Latest Situation of the COVID-19 as of 24:00 on 7 April 2020. Health Emergency Office. Available online: http:/ / www.nhc.gov.cn/xcs/yqtb/202004/5e2b6f0bd47d48559582242e3878447d. shtml (accessed on 8 April 2020).

6. Sohu News. Decisive Battle against SARS. Available online: http://news.sohu.com/57/26/subject206252657.shtml (accessed on 25 June 2003). 
7. Gorbalenya, A.E.; Baker, S.C.; Baric, R.S.; de Groot, R.J.; Drosten, C.; Gulyaeva, A.A.; Haagmans, B.L.; Lauber, C.; Leontovich, A.M.; Neuman, B.W.; et al. The species Severe acute respiratory syndrome-related coronavirus: Classifying 2019-nCoV and naming it SARS-CoV-2. Nat. Microbiol. 2020, 5, 536-544. [CrossRef]

8. Guan, W.-J.; Ni, Z.-Y.; Hu, Y.; Liang, W.-H.; Ou, C.-Q.; He, J.-X.; Liu, L.; Shan, H.; Lei, C.-L.; Hui, D.S. Clinical characteristics of coronavirus disease 2019 in China. N. Engl. J. Med. 2020, 382, 1708-1720. [CrossRef]

9. Yang, Z.; Zeng, Z.; Wang, K.; Wong, S.-S.; Liang, W.; Zanin, M.; Liu, P.; Cao, X.; Gao, Z.; Mai, Z. Modified SEIR and AI prediction of the epidemics trend of COVID-19 in China under public health interventions. J. Thorac. Dis. 2020, 12, 165. [CrossRef] [PubMed]

10. Sun, T.; Wang, Y. Modeling COVID-19 epidemic in Heilongjiang province, China. Chaos Solitons Fractals 2020, $138,109949$. [CrossRef] [PubMed]

11. Desjardins, M.; Hohl, A.; Delmelle, E. Rapid surveillance of COVID-19 in the United States using a prospective space-time scan statistic: Detecting and evaluating emerging clusters. Appl. Geogr. 2020, 118, 102202. [CrossRef] [PubMed]

12. Carletti, T.; Fanelli, D.; Piazza, F. COVID-19: The unreasonable effectiveness of simple models. Chaos Solitons Fractals 2020, 5, 100034. [CrossRef]

13. Sun, Z.; Zhang, H.; Yang, Y.; Wan, H.; Wang, Y. Impacts of geographic factors and population density on the COVID-19 spreading under the lockdown policies of China. Sci. Total Environ. 2020, 746, 141347. [CrossRef]

14. Anderson, R.M.; May, R.M. Infectious Diseases of Humans: Dynamics and Control; Oxford University Press: New York, NY, USA, 1991.

15. Lekone, P.E.; Finkenstädt, B.F. Statistical inference in a stochastic epidemic SEIR model with control intervention: Ebola as a case study. Biometrics 2006, 62, 1170-1177. [CrossRef]

16. Biswas, M.H.A.; Paiva, L.T.; De Pinho, M. A SEIR model for control of infectious diseases with constraints. Math. Biosci. Eng. 2014, 11, 761. [CrossRef]

17. Fu, S.; Milne, G. Epidemic modelling using cellular automata. In Proceedings of the Australian Conference on Artificial Life, Canberra, Australia, 6-7 December 2003.

18. Ahmed, E.; Agiza, H. On modeling epidemics including latency, incubation and variable susceptibility. Phys. A 1998, 253, 347-352. [CrossRef]

19. Mikler, A.R.; Venkatachalam, S.; Abbas, K. Modeling infectious diseases using global stochastic cellular automata. J. Biol. Syst. 2005, 13, 421-439. [CrossRef]

20. Wolfram, S. Cellular automata as models of complexity. Nature 1984, 311, 419-424. [CrossRef]

21. White, R.; Engelen, G. High-resolution integrated modelling of the spatial dynamics of urban and regional systems. Comput. Environ. Urban Syst. 2000, 24, 383-400. [CrossRef]

22. Wilensky; NetLogo. Center for Connected Learning and Computer-Based Modeling (CCL). Available online: http://ccl. northwestern.edu/netlogo/ (accessed on 7 April 2020).

23. Wuhan Headquarters for COVID-19 Prevention and Control. Notice of Wuhan Headquarters for COVID-19 Prevention and Control (No. 1). Available online: http:/ / www.gov.cn/xinwen/2020-01/23/content_5471751.htm (accessed on 23 January 2020).

24. Hubei Province Headquarters for COVID-19 Prevention and Control. Notice of Hubei Province Headquarters for COVID-19 Prevention and Control. Available online: http:/ /www.hubei.gov.cn/zhuanti/2020/gzxxgzbd/zxtb/202004/t20200407_2207131. shtml (accessed on 7 April 2020).

25. Liu, H.; Jiang, D.; Yang, X.; Luo, C. Spatialization approach to $1 \mathrm{~km}$ grid GDP supported by remote sensing. Geo-Inform. Sci. 2005, $2,120-123$.

26. Tian, G.; Ouyang, Y.; Quan, Q.; Wu, J. Simulating spatiotemporal dynamics of urbanization with multi-agent systems-A case study of the Phoenix metropolitan region, USA. Ecol. Model. 2011, 222, 1129-1138. [CrossRef]

27. Liu, D.; Zheng, X.; Wang, H. Land-use Simulation and Decision-Support system (LandSDS): Seamlessly integrating system dynamics, agent-based model, and cellular automata. Ecol. Model. 2020, 417, 108924. [CrossRef]

28. Cao, S.; Feng, P.; Shi, P. Study on the epidemic development of COVID-19 in Hubei province by a modified SEIR model. J. Zhejiang Univ. 2020, 49, 178-184.

29. Tang, B.; Xia, F.; Tang, S.; Bragazzi, N.L.; Li, Q.; Sun, X.; Liang, J.; Xiao, Y.; Wu, J. The Evolution of Quarantined and Suspected Cases Determines the Final Trend of the 2019-nCoV Epidemics Based on Multi-Source Data Analyses. Available online: https: / / papers.ssrn.com/sol3/papers.cfm?abstract_id=3537099 (accessed on 9 February 2020).

30. Wang, X.; Tang, S.; Chen, Y.; Feng, X.; Xiao, Y.; Xu, Z. When will be the resumption of work in Wuhan and its surrounding areas during COVID-19 epidemic? A data-driven network modeling analysis (in Chinese). Sci. Sin. Math. 2020, 50, 969-978. [CrossRef]

31. Wu, X.; Wang, Z.; He, Z.; Li, Y.; Wu, Y.; Wang, H.; Liu, Y.; Hao, F.; Tian, H. A follow-up study shows no new infections caused by patients with repeat positive of COVID-19 in Wuhan. medRxiv 2020. [CrossRef] 